\title{
Changes in adolescent mental and somatic health complaints throughout the COVID-19 pandemic: A three-wave prospective longitudinal study
}

Gertrud Sofie Hafstad ${ }^{\mathrm{a}, *}$, Sjur Skjørshammer Sætren ${ }^{\mathrm{a}, \mathrm{b}}$, Tore Wentzel-Larsen, and Else-Marie Augusti ${ }^{\mathrm{a}}$

${ }^{a}$ Norwegian Centre for Violence and Traumatic Stress Studies

${ }^{\mathrm{b}}$ CAMHS Sola, Division of Psychiatry, Stavanger University Hospital, Norway

*Corresponding author: Gertrud Sofie Hafstad, PhD, Norwegian Centre for Violence and

Traumatic Stress Studies, Gullhaugveien 1-3, 0409 Oslo, Norway. Email: g.s.hafstad@nkvts.no;

Telephone: +4741609916 .

\section{Acknowledgements}

We would like to thank Carina Schjem Grimsgaard, Anine Forsberg, and Linn Eirin Aronsen Haugen from our data collection team. The Norwegian Directorate for Children, Adolescents and Family Matters (Bufdir) funded the research. The funding source played no role in study design, data collection, analysis, and interpretation, manuscript writing, or decision to submit the article for publication. 


\section{ABSTRACT}

Purpose: Measures taken to limit the spread of the COVID-19 may have had unintended consequences for the mental and somatic health of children and adolescents.

Methods: A nationwide three-wave survey in a representative sample of 12 to 16 years olds in Norway, with baseline data collected in February 2019 ( $n=9240$, 49\% girls) and follow-ups in June 2020 ( $n=3564$, 49\% girls) and June 2021 ( $n=2540$, 47\% girls). Linear mixed-effects models were used to estimate change and identify predictors thereof in mental and somatic health complaints.

Results: Following an initial stable trend from before the pandemic to the early phase, both mental health problems (predicted value at T1 0.56 [CI 0.55, 0.58], T1-T2 change -0.04 [ CI 0.07, -0.02], T2-T3 change 0.12 [ CI 0.09, 0.14] and somatic health complaints (predicted value at T1 0.59 [95\% CI 0.58, 0.61], T1-T2 difference -0.09 [95\% CI -0.11, -0.65], T2-T3 difference 0.18 [95\% CI 0.15, 0.21]) increased significantly 15 months into the pandemic, when controlling for age in the models. When compared to boys, girls had a significantly more pronounced increase in mental health problems and somatic health; loneliness in the early stages of the pandemic significantly predicted health complaints one year later, both mental and somatic complaints.

Conclusion: Our findings suggest that the prolonged situation of recurrent lockdowns have had an impact on adolescent health in general, and on the health of girls in particular. Much of the rise in mental and somatic health complaints can be attributed to an increase in loneliness.

\section{IMPLICATIONS AND CONTRIBUTION}

To the best of our knowledge, this longitudinal population-based study is one of the few adolescent self-report studies with a large representative baseline sample, allowing us to investigate changes in symptoms during the first and second pandemic years. The current evidence suggests that if societies continue to use frequent and persistent measures to combat the spread of COVID-19 virus, we can expect a continued appreciable increase in symptoms of mental and somatic health problems among adolescents. It is critical to continue monitoring mental and somatic health symptoms among adolescents in order to learn about the persistence of symptoms after the pandemic. 
Restrictions aimed at controlling the spread of the COVID-19 have had an impact on the lives of billions of children, adolescents, and their families[1]. Many people have experienced recurring episodes of social isolation as well as a prolonged fear of contagion. Other pandemicrelated factors have been discussed as potential risk factors for psychopathology during the pandemic, such as mandatory school closures, cancellation of all sports and leisure activities, financial hardship, and child and family stress associated with these mitigation measures [2-4]. Norway, a sparsely populated Northern European country known for its social democratic welfare state, was one of the first to implement a nationwide lockdown on March 12, 2020. This included the mandatory closure of all schools at all levels, as well as the cancellation of organized sports. In general, social distancing measures, such as limiting social interactions to household members, were strongly encouraged. While pre- and primary schools reopened within a few weeks, other educational institutions, as well as organized sports for adolescents, remained closed until mid-May 2020. As a result of the dynamic change in contagion, new restrictions have been implemented throughout 2020 and 2021. In Norway, adolescents have been disproportionately affected by restrictions when compared to younger children and adults, as middle and high schools faced the most restrictive measures, including repeated and extended home schooling periods and cancelled sports activities throughout the pandemic years. At the same time, adolescence may be regarded as a vulnerable developmental stage during which mental health issues emerge [5]. Based on previous and current knowledge about adolescent mental health and related risk factors, we are particularly concerned that ongoing efforts to combat the spread of the virus may amplify an already increasing trend in mental health problems among adolescents [6].

Concerns have been raised by the rapid accumulation of research aimed at understanding the impact of the pandemic on multiple domains of adolescent health. According to metaanalytic evidence from the first year of the pandemic, adolescent mental health has deteriorated globally [7]. Despite being less studied, emerging evidence indicates an increase in somatic health complaints [8-10]. Adolescents with pre-existing mental and/or somatic health problems appear to be especially vulnerable to aggravating health problems during the pandemic [10]. However, the evidence from the first year of the pandemic in Norway indicates that most adolescents adapted efficiently to pandemic conditions, as evidenced by no notable changes in depressive symptoms, somatic health, or friendships compared to pre-pandemic levels $[11,12]$. 
The inconclusive results from existing studies may be attributed to factors such as variations in societies' pandemic-related burdens, timing of data collection during the pandemic, and study design [13]. For example, the timing of data collection during the pandemic may have impact on findings, as symptoms may vary as pandemic measures are implemented [3]. During the pandemic, the majority of studies with adolescent samples published to date have been crosssectional, relying primarily on convenience and online-based non-probability sampling [13]. However, studies with adolescents must account for the wide age and gender differences in mental health problems during adolescence [14]. As demonstrated by our first study on changes in Norwegian adolescents' mental health during the pandemic, the observed increase in symptoms appeared to be driven by age rather than pandemic-related factors [12]. Continuous prospective investigation of changes in symptoms is required to understand the long-term changes in symptoms during several waves of infection and subsequent measures. Preferably, a pre-pandemic sample would be followed over time.

To promote adolescent resilience and mitigate the negative effects of current and future pandemics, we must first identify the factors associated with the development of mental and somatic health problems in adolescents during the pandemic. We aimed to determine 1) whether mental health problems, in terms of symptoms of anxiety and depression, and somatic health complaints increased or decreased from before the pandemic to one year into the pandemic, 2) whether certain groups of adolescents were disproportionately affected by the pandemic, as evidenced by a greater increase in symptoms, and 3) what factors predicted higher levels of mental and somatic health complaints one year into the pandemic.

\section{Methods}

\section{Design and sample}

The study is part of an ongoing nationwide longitudinal survey study of a representative sample of 9,240 Norwegian 12-to-16-year-olds[15]. The first wave of the survey was conducted in February 2019, about a year before the COVID-19 outbreak, with two follow-up assessments in June 2020 and June 2021, respectively, to assess the potential impact of the COVID-19 pandemic on adolescent health and wellbeing. The survey was administered in schools sampled for their geographical representativeness (all regions of Norway were covered), school size, and ethnic minority status. Students participated by filling out an online questionnaire. The survey 
was only available in Norwegian, and students who did not have a sufficient level of Norwegian language skills were unable to participate.

A total of 3,564 $(50.1 \%$ girls, $\mathrm{n}=1,776)$ took part in the second data collection wave (T2, June 2020), while 3,540 (47.3\% girls, $\mathrm{n}=1,675)$ took part in the third data collection wave (T3, June 2021). Age range at all three data collection points was 12-16 years. Figure 1 depicts an overview of the study design and data collection points.

\section{Figure 1 here.}

\section{Attrition and representativeness}

In terms of all included background variables, samples from T1, T2 and T3 were comparable (Table 1). However, further attrition analyses revealed that those who participated at all three time points reported fewer symptoms of mental health problems (mean $=.42, \mathrm{SD}=.53$, possible range 0-3) compared to those who only participated at T1 (mean $=.59, \mathrm{SD}=67 ; p<$ .001). Similarly, participants who participated at all three time points reported fewer symptoms of somatic health complaints (mean $=.49, \mathrm{SD}=.53$ ) than those who only participated at $\mathrm{T} 1$ (mean $=.60, \mathrm{SD}=68 ; p=.001)$. This suggests that adolescents with higher levels of mental health problems and somatic health complaints at T1 were less likely to participate in follow-up surveys at $\mathrm{T} 2$ or $\mathrm{T} 3$.

\section{Measures}

Background variables: Participants reported their age in years at all three time points. Perceived family affluence was measured using two questions: whether the adolescent perceived the family as having sufficient economic means to purchase necessary goods, and whether the adolescent had experienced a decline in participation in after-school activities due to family finances. We also assessed the parents' country of origin, which was coded as Norway, another Nordic country, Europe (outside the Nordic region), or outside of Europe. A history of abuse was evaluated using a series of questions that assessed lifetime exposure to physical, emotional, or sexual abuse, as well as witnessing domestic violence. These experiences were combined into a cumulative scale (possible range 0-7), with a higher score indicating greater exposure. 
Anxiety and depression symptoms: We examined anxiety and depression symptoms using the Hopkins Symptom Checklist-10 (HSCL-10) at all three time points. HSCL-10 is a self-report questionnaire used in population surveys to assess psychological distress. Each item is presented on a four-point scale (i.e., 0-3), ranging from 'Not at all' and 'A little' to 'Quite a bit' and 'Extremely', and is summarized as a mean score that requires a valid response on at least half of the items in order to compute a score. Items covered symptoms of worry, anxiety and lack of interest (e.g., "Feeling tense or keyed up", "Suddenly scared for no reason", "Feeling blue"). In Norwegian primary care institutions, the HSCL-10 is used as a valid instrument for screening and identifying depression in adolescents aged 14 to 16 years [16] Somatic symptoms: At all three time points, a brief version of the Children's Somatic Symptoms Inventory CSSI $[17,18]$ was used to assess self-reported somatic symptoms. Items with adequate statistical properties from the validation study were selected in collaboration with the authors of the CSSI-24 [17]. The scale includes eight items covering stomach pain, headache, back pain, arm or leg pain, faintness or dizziness, rapid heartbeat, nausea, and fatigue All items are presented on four-point scale ranging from 0 (= not bothered) to 3 (= very much bothered). A mean score was calculated when at least $50 \%$ of the items had a valid response, and internal consistency for the scale was .827.

Pandemic-related worries: With a set of eight questions modified from the Pandemic Anxiety Scale, we inquired about specific concerns about the pandemic and possible consequences at $\mathrm{T} 2$ and T3 [19]. Each item was rated on a 5-point Likert scale ranging from 0 ('Strongly disagree') to 4 ('Strongly agree').

Loneliness: Adolescents' self-reported loneliness over the previous two weeks was mapped using the UCLA loneliness scale short form with three items at T2 and T3 assessments [20,21]. The three-item UCLA version has been shown in large-scale studies to be accurate in measuring loneliness and is related to objective self-isolation [21]. The questions were rated on a threepoint scale ranging from 1 ('Never') to 3 ('Always'), and a composite mean score was calculated for the three items.

\section{Procedures}

The UEVO-study [28] included 70 schools of which subsamples accepted invitations to participate in T2 and T3. Schools were approached and asked to administer the web-based 
survey to their entire student body, grades 8 through 10. The survey was administered during school hours by the schools that agreed to participate. The web-based survey took approximately 40 minutes at T1, 15 minutes at T2, and 30 minutes at T3 to complete after watching a short animated video about the study and the ethical principles of voluntary participation, confidentiality, and the right to withdraw at any time during the study without having to give a reason. Students completed the survey in class using a PC, tablet, or cell phone.

\section{Ethics}

The study protocol was approved by the Regional committee for ethics in medical and health research in the Southeastern region of Norway (Case \#2018/522). All participants gave informed written consent, and the adolescents did not need parental permission to participate.

\section{Data analyses}

Descriptive statistics for background variables and key outcome variables are presented as percentages, means, and standard deviations. The sample characteristics are presented for all participants who participated at each time point, as well as for participants who participated at all three time points (the longitudinal sample).

To assess the potential impact of the pandemic and lockdown, we ran two mixed effects models, estimating change and predictors of change in 1) anxiety and depression symptoms, and 2) somatic health complaints. Time was treated as a categorical variable with three levels in the mixed effects models. The models included a number of background variables with an emphasis on their importance for change in the dependent variables, and thus only variables most likely present prior to the first assessment (T1) were included so that the temporal interaction was not hampered. These were gender, low family affluence, family ethnic minority status, and a history of violence exposure. Because mental health problems increase with age throughout adolescence, we controlled for age in the models.

The mixed effects models permitted the use of all observed data, regardless of whether individuals had observations at all time points. A positive time coefficient from the mixed-effects model indicates worsening in mental health problems or somatic health complaints associated with the pandemic. To investigate heterogeneity in the effect of the pandemic, interactions between time (before to during the pandemic) and the five risk variables were fitted. In general, missing data in outcome variables were handled by applying the half rule, i.e. only participants 
with valid observations on at least half of the items were included in the scale scores. Under the less restrictive missing at random assumption, mixed effects models provide valid inference with missing data in the dependent variable.

In the final step, variables predicting levels of mental health problems and somatic health complaints at T3 were analyzed. Two hierarchical multiple regression analyses are presented, investigating variables predicting mental health problems and somatic health complaints, respectively, one year into the pandemic (T3). The dependent variable's distribution was investigated, and to account for non-normality in variables, both regression models were bootstrapped with 1000 replications, yielding confidence intervals for estimates that did not rely on specific distributional assumptions.

Descriptive statistics and linear regression models were conducted in SPSS version 26 (IBM Corp, released 2019), while mixed effects models were run using the nlme (linear and nonlinear mixed effects models) package in $\mathrm{R}$ ( $\mathrm{R}$ Core Team, 2020).

\section{Results}

Table 1 provides an overview of sample characteristics.

\section{Table 1 here}

\section{Changes in mental and somatic health complaints over time}

We fitted two mixed effects models, one for anxiety and depression and one for somatic health complaints, one year before the pandemic (2019), three months into the first outbreak (2020), and one year later (2021) to investigate the evolution of mental and somatic health complaints over time. Table 2 shows the parameter estimates for the fixed and random effects. The basic model estimating the change in mental health problems in the total sample, showed a non-linear development over time, reflecting a flat trend from 2019 to 2020, followed by an increase in symptoms from 2020 to 2021 (Predicted value at T1 0.56 [95\% CI 0.55,0.58], T1-T2 difference -0.04 [95\% CI -0.07,-0.02], T2-T3 difference 0.12 [95\% CI 0.09,0.14]). As shown in Table 2, Model 1 with covariates assessed at T1 in 2019 (sex, age, family affluence, country of origin, single-parent household, a history of violence exposure) revealed that, while all covariates were associated with significantly higher levels of anxiety and depression one year 
prior to the pandemic, girls had a significantly larger increase in mental health symptoms before the pandemic and throughout the period compared to boys.

\section{Table 2 here}

A strikingly similar trend was observed for somatic health complaints throughout the pandemic. The basic model, which estimated the change in somatic health complaints in the total sample while controlling for increase in age, also revealed a flat trend from 2019 to 2020, followed by an increasing trajectory of somatic health complaints from 2020 to 2021 (Predicted value at T1 0.59 [95\% CI 0.58, 0.61], T1-T2 slope -0.09 [95\% CI -0.11, -0.65], T2-T3 slope 0.18 [95\% CI 0.15, 0.21]). As shown in Table 2, Model 2 with covariates assessed at T1 in 2019 revealed that girls experienced a significantly greater increase in somatic health complaints from prior to the pandemic and throughout the period than boys.

\section{Predicting mental and somatic health complaints one year into the pandemic}

Tables 3 and 4 show the results of two multiple hierarchical regression models that predict mental and somatic complaints at T3. According to model 3 (Table 4), pre-pandemic mental health problems, female gender, living in a single-parent household, and abuse exposure significantly predicted higher mental health scores at T3, even when pandemic-related risk factors were controlled for at T2 (model 2) and T3 (model 3). Furthermore, loneliness at T2 was associated with higher mean scores on HSCL at T3. Pandemic-related worries, on the other hand, were inversely related to mean scores on HSCL at T3, indicating that higher pandemic-related worries at T2 predicted lower scores on HSCL at T3. Loneliness at T3 was also significantly related to mean scores on HSCL at T3 when controlling for concurrent and pre-pandemic risk factors (model 3). As shown in Table 4, we found somewhat similar results for somatic health complaints one year into the pandemic, except that living in a single-parent household and having pandemic-related worries assessed at T2 or T3 did not significantly predict scores on CSSI at T3. The models explained $40 \%$ of the variance in anxiety depression at T3 and $33 \%$ of the variance in somatic health complaints at $\mathrm{T} 3$.

\section{Table 3 here}




\section{Table 4 here}

\section{Discussion}

Using a nationwide prospective longitudinal study of Norwegian adolescents from before to during the pandemic, we investigated multiple aspects of adolescent self-reported mental and somatic health complaints. We discovered that one year (15 months) into the pandemic, after an initial stable trend from before the pandemic to the early phase of the pandemic, both mental and somatic health complaints increased. The increase in symptoms from June 2020 to June 2021 was significant both for mental and somatic health complaints. Girls appeared to be disproportionately affected during this time period, as evidenced by a significantly higher increase in both mental and somatic health complaints when compared to boys. Loneliness in the early stages of the pandemic was a significant contributing factor to higher levels of health complaints one year into the pandemic; both for mental and somatic complaints. Prior abuse exposure and living in a single-parent household prior to the pandemic outbreak were also associated with elevated symptoms of mental health problems. A history of abuse was also linked to an increase in somatic health complaints. However, and contrary to expectations, pandemic-related concerns were found to be inversely related to symptoms of mental health problems.

The observed increase in anxiety and depression, as well as in somatic complaints, one year into the pandemic, highlights the concern that prolonged and repeated measures may have had a negative impact on the adolescent population in terms of increased risk for both mental and somatic health complaints [10]. So far, findings from Norway and other developed countries on changes in symptoms of mental and somatic health have been inconclusive, and few studies have looked at changes in symptoms over a longer period of time. Our findings suggest that if we continue to implement frequent and consistent measures to combat the spread of the COVD-19 virus, we can expect an increase in symptoms of mental and somatic health complaints among adolescents.

In line with previous research on mental and somatic health during the COVID-19 pandemic [14], we discovered that adolescent girls had a relatively greater increase in symptoms of both mental and somatic health complaints than boys. In addition to having more symptoms at the start, girls showed a trend throughout the pandemic that was characterized by a significant 
worsening of mental and somatic health symptoms when compared to boys. Furthermore, greater changes in loneliness for girls compared to boys during the pandemic may explain a relatively increased risk for internalizing symptoms. As a result, we can speculate that adolescent girls' mental and somatic well-being may be more dependent on social activities that are restricted during lock down than boys'. Systematic reviews and meta-analytic findings suggest that adolescent girls may be especially vulnerable to anxiety and depression during the pandemic $[7,22]$. On an important note, we assessed symptomatology, which is more commonly reported by girls. Boys, on the other hand, may be more prone to externalizing psychopathology, which we did not examine in this study.

According to our findings after the first wave of the pandemic, children in single-parent households experienced an increase in mental health symptoms. Surprisingly, our findings suggest that pandemic-related worries may buffer symptoms of depression and anxiety, accounting for other risk factors. Despite the small estimates, the inverse relationship could represent an adaptive coping process rather than a maladaptive response to the pandemic as a collective threat. In line with this, increased anxiety during the pandemic has been found to promote protective behaviors [23].

In the present study, increased loneliness during the first year of the pandemic was strongly related to elevated symptoms of both mental and somatic complaints. The crosssectional and longitudinal association between loneliness and mental health problems in adolescents is well established in the literature [24], and the relationship between loneliness and mental health problems is most likely bidirectional [25]. However, in line with a recent metaanalysis of 80 studies on loneliness in adolescents, we discovered that loneliness was associated with subsequent anxiety and depression [24]. Our findings add to previous research by demonstrating that initial high levels of loneliness are predictors of correspondingly high levels of internalizing symptoms, both in terms of mental and somatic health complaints.

\section{Strengths and limitations}

The sample's representativeness, as well as the prospective design that allows us to compare adolescents' mental and somatic health complaints before and during the COVID-19 pandemic (June 2020 and 2021), are clear strengths of this study. 
School-based surveys, such as this one, frequently fail to include more marginalized groups, such as adolescents with psychosocial problems, adolescents from less affluent or less educated backgrounds, and adolescents who drop out of school. As a result, there is always the risk that findings will underestimate the difficulties faced by the entire population. Attrition analyses revealed that, while the sample that participated in all measurements was overall comparable to those who dropped out, participants with higher symptoms of mental health problems and somatic complaints at T1 dropped out at T2 and T3. As a result, we may have underestimated the pandemic's impact on these measures.

\section{Implications and conclusion}

Although the trend of increasing mental and somatic health complaints in adolescents is small, it is concerning because it shows a clear acceleration over the last year, and thus suggests that the prolonged pandemic-related restrictions have taken a toll on the adolescent population. Continuous monitoring of symptoms in representative and diverse samples of adolescents must be prioritized, to obtain a knowledge-based ground for developing youth mental health interventions. Efforts should be made to reach out to the general youth population with interventions aimed at reducing loneliness and enhancing social relationships in times of crises. Adolescent pandemic mitigation measures must be kept to a bare minimum.

\section{Contributors}

GSH and EMA designed the study, were responsible the acquisition of funding, and were in charge of data collection. GSH, TWL, and SSS carried out all data analyses and produced the tables. SSS conducted and summarized the systematic literature search, and conducted the attrition analyses. GSH wrote the first draft of the manuscript and all authors commented on and edited the final version.

\section{Declaration of interests}

We declare no competing interests.

\section{Data sharing}


The dataset is administrated by the Norwegian Centre for Violence and Traumatic Stress Studies. Approval from a Norwegian regional committee for medical and health research ethics (https://helseforskning.etikkom.no) is a pre-requirement. Guidelines for access to the data are found at the longitudinal cohort website uevo.nkvts.no.

\section{References}

[1] Brooks SK, Webster RK, Smith LE, et al. The psychological impact of quarantine and how to reduce it: rapid review of the evidence. Lancet 2020;395:912-920.

[2] Holmes EA, O'Connor RC, Perry VH, et al. Multidisciplinary research priorities for the COVID-19 pandemic: a call for action for mental health science. Lancet Psychiatry 2020;7:547-560.

[3] Creswell C, Shum A, Pearcey S, et al. Young people's mental health during the COVID-19 pandemic. Lancet Child Adoles Health 2021;5:535-37.

[4] Hafstad GS, Augusti E-M. A lost generation? COVID-19 and adolescent mental health. Lancet Psychiatry 2021

[5] De Girolamo G, Dagani J, Purcell R, et al. Age of onset of mental disorders and use of mental health services: needs, opportunities and obstacles. Epidemio Psychiatr Sci 2012;21:47.

[6] Blomqvist I, Henje Blom E, Hägglöf $B$, et al. Increase of internalized mental health symptoms among adolescents during the last three decades. Eur J Public Health 2019;29:925-31.

[7] Racine N, McArthur BA, Cooke JE, et al. Global prevalence of depressive and anxiety symptoms in children and adolescents during COVID-19: a meta-analysis. JAMA Pediatr 2021

[8] Liu S, Liu Y, Liu Y. Somatic symptoms and concern regarding COVID-19 among Chinese college and primary school students: A cross-sectional survey. Psychiatry Res 2020;289:113070.

[9] Ravens-Sieberer U, Erhart M, Rajmil L, et al. Reliability, construct and criterion validity of the KIDSCREEN-10 score: a short measure for children and adolescents' well-being and healthrelated quality of life. Qual Life Res 2010;19:1487-500. doi: 10.1007/s11136-010-9706-5 [published Online First: 2010/07/30]

[10] Zijlmans J, Teela L, van Ewijk H, et al. Mental and social health of children and adolescents with preexisting mental or somatic problems during the COVID-19 pandemic lockdown. Front Psychiatry 2021;12

[11] Andreas JB, Brunborg GS. Self-reported mental and physical health among Norwegian adolescents before and during the COVID-19 pandemic. JAMA Netw Open 2021;4:e2121934-e34.

[12] Hafstad GS, Sætren SS, Wentzel-Larsen T, et al. Adolescents' symptoms of anxiety and depression before and during the Covid-19 outbreak-A prospective population-based study of teenagers in Norway. Lancet Reg Health Eur 2021;5:100093.

[13] Vaillancourt $T$, Brittain $H$, Krygsman $A$, et al. Assessing the quality of research examining change in children's mental health in the context of COVID-19. Univ Ottawa J Med 2021;11(1)

[14] Thorisdottir IE, Asgeirsdottir BB, Kristjansson AL, et al. Depressive symptoms, mental well-being and substance abuse among adolescents before andduring the COVID-19 pandemic in Iceland: a longitudinal analysis. The Lancet Psychiatry 2021 
[15] Hafstad GS, Sætren SS, Myhre MC, et al. Cohort profile: Norwegian youth study on child maltreatment (the UEVO study). BMJ Open 2020;10:e038655.

[16] Haavet OR, Sirpal MK, Haugen W, et al. Diagnosis of depressed young people in primary health care-a validation of HSCL-10. Family Practice 2010;28:233-37.

[17] Walker LS, Beck JE, Garber J, et al. Children's Somatization Inventory: psychometric properties of the revised form (CSI-24). J Pediatr Psychol 2009;34:430-40.

[18] Stone AL, Walker LS, Heathcote LC, et al. Somatic symptoms in pediatric patients with chronic pain: Proposed clinical reference points for the children's somatic symptoms inventory (formerly the children's somatization inventory). J Pain 2019;20:932-40.

[19] McElroy E, Patalay P, Moltrecht B, et al. Demographic and health factors associated with pandemic anxiety in the context of COVID-19. Br J Health Psychol 2020;25:934-944.

[20] Hays RD, DiMatteo MR. A short-form measure of loneliness. J Pers Assess 1987;51:69-81.

[21] Hughes ME, Waite $L$, Hawkley LC, et al. A short scale for measuring loneliness in large surveys: Results from two population-based studies. Res Aging 2004;26:655-72.

[22] Ma L, Mazidi M, Li K, et al. Prevalence of mental health problems among children and adolescents during the COVID-19 pandemic: A systematic review and meta-analysis. J Affect Disord 2021

[23] Salali GD, Uysal MS, Bevan A. Adaptive function and correlates of anxiety during a pandemic. 2021

[24] Loades ME, Chatburn E, Higson-Sweeney N, et al. Rapid systematic review: the impact of social isolation and loneliness on the mental health of children and adolescents in the context of COVID-19. J Am Acad Child Adolesc Psychiatry 2020;59:1218-39. e3.

[25] Lasgaard M, Goossens L, Elklit A. Loneliness, depressive symptomatology, and suicide ideation in adolescence: Cross-sectional and longitudinal analyses. J Abnorm Child Psychol 2011;39:137-50. 


\section{Research in Context}

\section{Evidence before this study}

We searched PubMed, Psychinfo and Web of Science for peer-review papers published from January 1, 2020, to September 2020, with the language restricted to English. We paired the terms “mental health", “depression", “anxiety" or "PTSD” with "COVID-19” or "pandemic", and with "cross-sectional" or "longitudinal". These searches were restricted to the following population characteristics: "children", "adolescents" or "young adults". Additional papers were identified by checking relevant references on child and adolescent mental health during COVID-19. We identified 110 relevant papers, including 22 studies with a longitudinal design and pre-data. Most studies included small samples via convenience sampling, and included measures of symptoms only within the first year into lock down. There was a lot of methodological diversity and geographical variation among the studies. The results were mixed, showing diverse impacts on adolescents' mental health, depending on prepandemic mental health and concurrent risk factors related to sociodemographic backgrounds. Although there was a trend toward an increase in mental health problems, some studies suggest that higher levels corresponded to periods of peak infection rates and lock-down strategies.

Follow-up studies that rely on convenience samples may be biased towards less change in symptoms because study attrition is more likely among adolescents with poor functioning. 
Figure 1. Overview of study design and sample size at the three data collection points

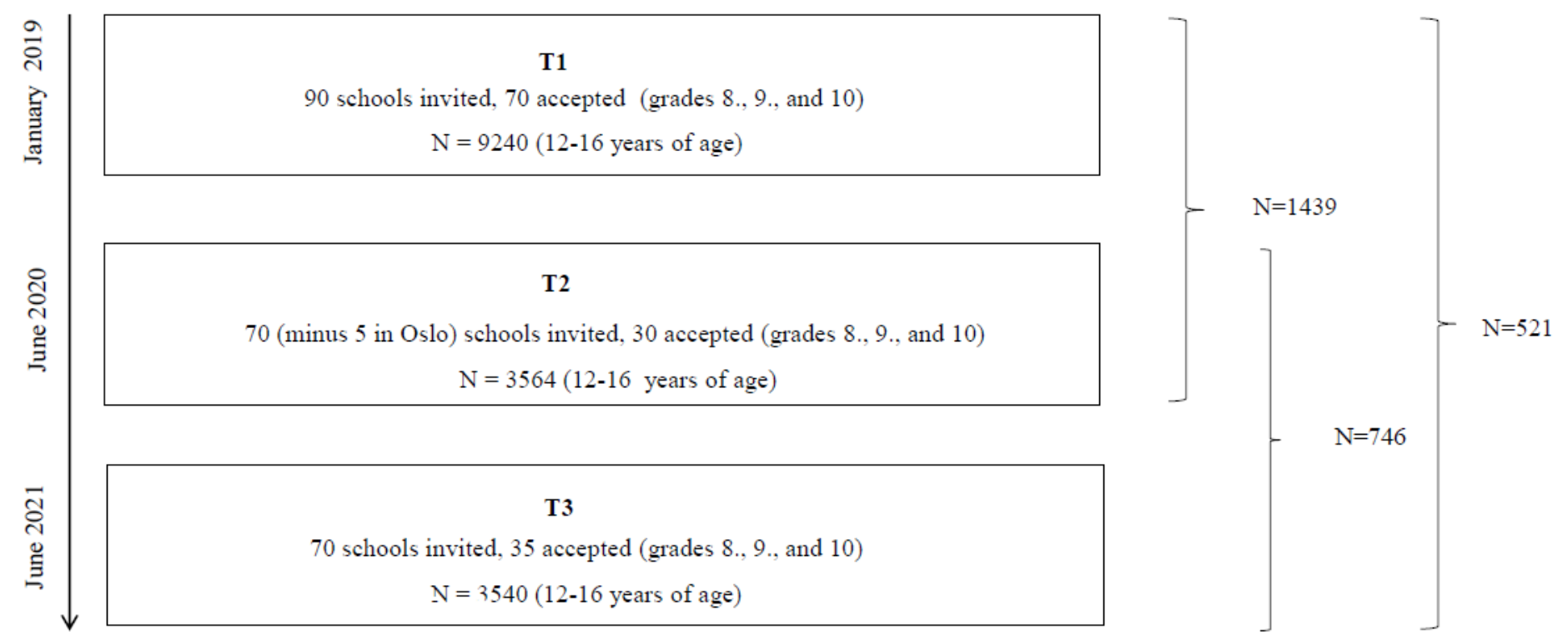


Table 1. Characteristics of the sample at the three measurement points

\begin{tabular}{|c|c|c|c|c|c|c|c|c|}
\hline & \multirow{2}{*}{\multicolumn{2}{|c|}{$\begin{array}{l}\text { T1 (2019) } \\
\mathrm{N}=9240\end{array}$}} & \multirow{2}{*}{\multicolumn{2}{|c|}{$\begin{array}{c}\mathrm{T} 2(2020) \\
\mathrm{N}=3564\end{array}$}} & \multirow{2}{*}{\multicolumn{2}{|c|}{$\begin{array}{c}\text { T3 (2021) } \\
\text { N=3540 }\end{array}$}} & \multirow{2}{*}{\multicolumn{2}{|c|}{$\begin{array}{c}\text { T1_T2-T3* } \\
\text { N=521 }\end{array}$}} \\
\hline & & & & & & & & \\
\hline & $\mathrm{N}$ & $\%$ & $\mathrm{~N}$ & $\%$ & $\mathrm{~N}$ & $\%$ & $\mathrm{~N}$ & $\%$ \\
\hline \multicolumn{9}{|l|}{ Sex } \\
\hline Girls & 4592 & 50.0 & 1774 & 50.1 & 1675 & 47.6 & 254 & 51.0 \\
\hline Boys & 4538 & 49.4 & 1733 & 49.0 & 1733 & 49.0 & 241 & 48.4 \\
\hline Non binary & 63 & 0.7 & 33 & 0.9 & 63 & 1.8 & $<5$ & - \\
\hline \multicolumn{9}{|l|}{ Single parent household } \\
\hline Yes & 2725 & 29.7 & 1070 & 30.3 & 1077 & 30.7 & 138 & 27.8 \\
\hline No & 6458 & 70.3 & 2457 & 69.7 & 2429 & 69.3 & 358 & 72.2 \\
\hline \multicolumn{9}{|l|}{ Country of origin } \\
\hline Non-Nordic & 2349 & 25.9 & 858 & 24.6 & 904 & 26.1 & 101 & 20.5 \\
\hline Nordic & 6707 & 74.1 & 2627 & 75.4 & 2554 & 73.9 & 391 & 79.5 \\
\hline \multicolumn{9}{|l|}{ Family affluence } \\
\hline Low & 351 & 3.8 & 103 & 3.0 & 131 & 3.8 & 10 & 2.0 \\
\hline High/ Normal & 8773 & 96.2 & 3369 & 97.0 & 3337 & 3.8 & 484 & 98.0 \\
\hline Age: mean, SD (12-16) & 14.11 & 0.8 & 14.52 & 0.9 & 14.48 & 0.96 & 14.53 & 0.52 \\
\hline HSCL: mean, SD (0-3) & 0.57 & 0.66 & 0.55 & 0.64 & 0.72 & 0.74 & 0.42 & 0.53 \\
\hline CSSI: mean, SD (0-3) & 0.60 & 0.67 & 0.53 & 0.65 & 0.79 & 0.83 & 0.49 & 0.54 \\
\hline
\end{tabular}

*Sample with data on all three measurement points - descriptive data reported from the T1 measurement for comparison 
Table 2. Mixed effects model predicting change in anxiety depression (Model 1) and somatic health complaints (Model 2) from pre to early and late pandemic

\begin{tabular}{lrrrrrr}
\hline Model 1. Anxiety and depression & \multicolumn{3}{c}{ T2 } & & \multicolumn{2}{c}{ T3 } \\
\hline Interactions with time & Est. & \multicolumn{1}{c}{$95 \%$ CI } & p-value & Est. & $95 \%$ CI & p-value \\
\hline Gender (ref· boy) & -0.11 & $-0.21,0.01$ & $<.001$ & -0.17 & $-0.25,-0.09$ & $<.001$ \\
Family affluence (ref· low) & -0.08 & $-0.49,0.33$ & 0.69 & 0.06 & $-0.20,0.32$ & 0.66 \\
Living in the capital area of Norway & 0.01 & $-0.10,0.12$ & 0.85 & 0.04 & $-0.09,0.12$ & 0.40 \\
Parents' country of origin (ref·Nordic) & -0.00 & $-0.13,0.13$ & 0.97 & 0.00 & $-0.01,0.10$ & 0.94 \\
Single-parent household & 0.09 & $-0.03,0.21$ & 0.15 & 0.09 & $-0.01,0.18$ & 0.07 \\
Prior abuse exposure & -0.05 & $-0.11,0,00$ & 0.06 & -0.59 & $-0.10,0.02$ & 0.08 \\
\hline Model 2. Somatic health complaints & & T2 & & & T3 & \\
\hline Interactions with time & Est. & $95 \%$ CI & p-value & Est. & $95 \%$ CI & p-value \\
\hline Gender (ref· boy) & -0.16 & $-0.27,-0.04$ & $<.01$ & -0.23 & $-0.32,-0.13$ & $<.001$ \\
Family affluence (ref· low) & -0.00 & $-0.45,0.45$ & 0.99 & -0.07 & $-0.36,0.22$ & 0.61 \\
Living in the capital area of Norway & -0.01 & $-0.13,0.11$ & 0.86 & 0.01 & $-0.10,0.08$ & 0.80 \\
Parents' country of origin (ref Nordic) & -0.08 & $-0.07,0.22$ & 0.28 & 0.05 & $-0.07,0.16$ & 0.41 \\
Single-parent household & 0.06 & $-0.07,0.19$ & 0.36 & 0.04 & $-0.06,0.14$ & 0.43 \\
Prior abuse exposure & -0.04 & $-0.10,0.02$ & 0.14 & -0.05 & $-0.10,-0.00$ & 0.03 \\
\hline
\end{tabular}

Table 3. Linear regression model predicting the level of mental health complaints one year into the pandemic

\begin{tabular}{ccccccccc}
\hline Model 1 & \multicolumn{4}{c}{ Model 2 } & \multicolumn{3}{c}{ Model 3 } \\
\hline$B(95 \% \mathrm{CI})$ & $\beta$ & $P$ & $B(95 \% \mathrm{CI})$ & $\beta$ & $P$ & $B(95 \% \mathrm{CI})$ & $\beta$ & $P$ \\
\hline
\end{tabular}




\begin{tabular}{|c|c|c|c|c|c|c|c|c|c|}
\hline \multicolumn{10}{|l|}{ T1 variables } \\
\hline HSCL & $.41(.29, .53)$ & .32 & $<.001$ & $.29(.18, .40)$ & .23 & $<.001$ & $.27(.16, .37)$ & .21 & $<.001$ \\
\hline Male gender & $-.29(-.40,-.19)$ & -.23 & $<.001$ & $-.29(-.39,-.18)$ & -.22 & $<.001$ & $-.24(-.35,-.14)$ & -.19 & $<.001$ \\
\hline Family affluence & $-.35(-.79, .09)$ & -.07 & .121 & $-.38(-.80, .03)$ & -.07 & .071 & $-.36(-.75, .03)$ & -.07 & .070 \\
\hline $\begin{array}{l}\text { Non-nordic country } \\
\text { of origin }\end{array}$ & $.01(-.12, .15)$ & .01 & .833 & $.03(-.09, .16)$ & .02 & .600 & $.03(-.09, .15)$ & .02 & .638 \\
\hline $\begin{array}{l}\text { Single-parent } \\
\text { household }\end{array}$ & $.13(.01, .26)$ & .09 & .032 & $.14(.02, .26)$ & .09 & .020 & $.12(.01, .23)$ & .08 & .039 \\
\hline $\begin{array}{l}\text { Abuse exposure } \\
\text { (lifetime) }\end{array}$ & $.12(.05, .18)$ & .17 & .000 & $.12(.06, .18)$ & .18 & $<.001$ & $.09(.03, .15)$ & .14 & .002 \\
\hline \multicolumn{10}{|l|}{ T2 variables } \\
\hline Loneliness & & & & $.36(.26, .47)$ & .31 & $<.001$ & $.18(.07, .28)$ & .15 & .001 \\
\hline $\begin{array}{l}\text { Pandemic-related } \\
\text { worries }\end{array}$ & & & & $-.09(-.16,-.02)$ & -.10 & .014 & $-.08(-.17,-.00)$ & -.10 & .045 \\
\hline $\begin{array}{l}\text { Parents' pandemic- } \\
\text { related job loss }\end{array}$ & & & & $-.05(-.12, .03)$ & -.05 & .216 & $-.02(-.10,-.05)$ & -.03 & .496 \\
\hline \multicolumn{10}{|l|}{ T3 variables } \\
\hline Loneliness & & & & & & & $.38(.28, .48)$ & .34 & $<.001$ \\
\hline $\begin{array}{l}\text { Pandemic-related } \\
\text { worries }\end{array}$ & & & & & & & $.02(-.07, .11)$ & .02 & .689 \\
\hline $\begin{array}{l}\text { Parents' pandemic- } \\
\text { related job loss }\end{array}$ & & & & & & & $-.04(-.12, .04)$ & -.04 & .306 \\
\hline & $\mathrm{R}^{2}=.24$ & & & $\mathrm{R}^{2}=.33$ & & & $\mathrm{R}^{2}=.40$ & & \\
\hline
\end{tabular}


Table 4. Linear regression model predicting the level of somatic complaints one year into the pandemic

\begin{tabular}{|c|c|c|c|c|c|c|c|c|c|}
\hline & Model 1 & & & Model 2 & & & Model 3 & & \\
\hline & $B(95 \% \mathrm{CI})$ & $\beta$ & $P$ & $B(95 \% \mathrm{CI})$ & $\beta$ & $P$ & $B(95 \% \mathrm{CI})$ & $\beta$ & $P$ \\
\hline \multicolumn{10}{|l|}{ T1 variables } \\
\hline CSSI & $.55(.43, .68)$ & .39 & $<.001$ & $.53(.41, .65)$ & .38 & $<.001$ & $.50(.38, .62)$ & .35 & $<.001$ \\
\hline Male gender & $-.30(-.42,-.18)$ & -.20 & $<.001$ & $-.30(-.42,-.18)$ & -.21 & $<.001$ & $-.30(-.42,-.18)$ & -.21 & $<.001$ \\
\hline Family affluence & $-.11(-.60, .37)$ & -.02 & .655 & $-.13(-.60, .34)$ & -.02 & .587 & $-.16(-.62, .31)$ & -.03 & .511 \\
\hline $\begin{array}{l}\text { Non-nordic country } \\
\text { of origin }\end{array}$ & $-.00(-.15, .15)$ & .00 & .994 & $.01(-.13, .16)$ & .01 & .843 & $.00(-.14, .14)$ & .00 & .998 \\
\hline $\begin{array}{l}\text { Single-parent } \\
\text { household }\end{array}$ & $.11(-.03, .27)$ & .06 & .121 & $.10(-.03, .24)$ & .06 & .132 & $.10(-.03, .23)$ & .06 & .138 \\
\hline $\begin{array}{l}\text { Abuse exposure } \\
\text { (lifetime) }\end{array}$ & $.10(.03, .17)$ & .13 & .005 & $.09(.02, .14)$ & .12 & .008 & $.08(.01, .15)$ & .10 & .018 \\
\hline \multicolumn{10}{|l|}{ T2 variables } \\
\hline Loneliness & & & & $.23(.11, .34)$ & .17 & $<.001$ & $.11(-.01, .23)$ & .08 & .083 \\
\hline $\begin{array}{l}\text { Pandemic-related } \\
\text { worries }\end{array}$ & & & & $-.10(-.19,-.01)$ & -.10 & .021 & $-.03(-.13, .07)$ & -.03 & .546 \\
\hline $\begin{array}{l}\text { Parents' pandemic- } \\
\text { related job loss }\end{array}$ & & & & $.04(-.04, .13)$ & .04 & .325 & $.07(-.02, .15)$ & .06 & .131 \\
\hline
\end{tabular}

T3 variables 


$$
\mathrm{R}^{2}=.26
$$$$
\mathrm{R}^{2}=.30
$$$$
\mathrm{R}^{2}=.33
$$ 